\title{
Palliative care is everyone's business - is it yours, doctor?
}

To THE EDITOR: "Palliative care is everyone's business" is the theme of Palliative Care Australia's advocacy in 2013 and of Palliative Care Week, 19-25 May 2013. It is a timely and relevant slogan. Palliative care, once isolated in hospices for advanced cancer, is now a core medical discipline and a specialty established in major hospitals and universities, with community programs extending care to most advanced illness respiratory, cardiac, renal and neurological — and to aged care. 
"Everyone's business" aims first at the wider community, proclaiming the concern of palliative care as quality of living, not speeding of dying. Well managed care for patients with advanced and terminal illness creates its own publicity — attending family members say, "I want my death to be like that".

It is also relevant for health professionals, who say to the patient, "you are not ready for that, yet", as they try another intervention for serious illness. "Everyone's business" affirms management that is ready to refocus, frankly assess the best interests of the patient and explore relevant palliative options.

When palliative care becomes everyone's business, it will be featured in everyday media, in all health education, and offered throughout health care. The trials of ageing and advanced illness will be faced more squarely, with open discussion.

Advance care directives and the nomination of substitute medical decisionmakers remain little used. Conversation is more important than a written document. Open sharing to clarify values and hopes will help avoid futile measures, support best palliation and avoid unnecessary transfers to acute care, or futile lifesupport measures in emergency or intensive care.

The opportunity to die at home has wide community support. It allows family to travel with the patient along the final pathway. Families whose loved ones wish to die at home should seek this opportunity for them, private and public funding should support home care more strongly (it is usually cheaper), and providers should be challenged with the question: how often do you offer care at home for your terminally ill patients?

Improvement will see palliative care taught in undergraduate courses and extend to the years after graduation. Palliative care needs to be incorporated in the experience and practice of every generalist and specialist practitioner, becoming indeed "every doctor's business".
2 Palliative Care Australia, Canberra, ACT. Madd0064@flinders.edu.au

Competing interests: Yvonne Luxford is the CEO of Palliative Care Australia, which is the major promoter of Palliative Care Week.

doi: 10.5694/mjal3.10471

Ian Maddocks Emeritus Professor ${ }^{l}$

Yvonne Luxford Chief Executive Officer ${ }^{2}$

1 Flinders University, Adelaide, SA. 